\title{
Agreement between accelerometer-assessed and self-reported physical activity and sedentary time in colon cancer survivors
}

Terry Boyle ${ }^{1,2,3}$, Brigid M. Lynch ${ }^{4,5}$, Kerry S. Courneya $^{6}$, Jeff K. Vallance ${ }^{7}$

1. Cancer Control Research, British Columbia Cancer Agency, Vancouver, British Columbia, Canada

2. School of Population and Public Health, The University of British Columbia, Vancouver, British Columbia, Canada

3. Centre for Medical Research, The University of Western Australia, Perth, Western Australia, Australia

4. Physical Activity Laboratory, Baker IDI Heart and Diabetes Institute, Melbourne, Australia

5. School of Population and Global Health, The University of Melbourne, Melbourne, Australia

6. Behavioural Medicine Laboratory, Faculty of Physical Education and Recreation, University of Alberta, Edmonton, Canada

7. Faculty of Health Disciplines, Athabasca University, Athabasca, Canada

\section{Corresponding Author}

Terry Boyle

Cancer Control Research

BC Cancer Research Centre, BC Cancer Agency

675 West 10th Avenue

Vancouver BC V5Z 1L3 CANADA

Tel: (+ 1) 6046758066

E-mail: tboyle@bccrc.ca

Word Count: 2499

Tables: 2 


\begin{abstract}
Purpose: Research conducted on the general population indicates self-reported measures of physical activity and sedentary behaviour are inaccurate when compared with objective measures, however it is not clear if this also applies to cancer survivors. In this study we compared accelerometer-based and self-reported measures of moderate- to vigorous-intensity physical activity (MVPA) and sedentary time among colon cancer survivors.
\end{abstract}

Methods: A total of 176 colon cancer survivors, recruited from the Western Australia and Alberta cancer registries, wore an Actigraph GT3X+ accelerometer for seven days and completed self-reported questions about recent MVPA (Godin Leisure-Time Exercise Questionnaire) and usual sedentary time (Marshall Domain-Specific Sitting Questionnaire). Accelerometer data were processed using 60 second epochs and summarised using Freedson cutpoints. Spearman's rho and intraclass correlation coefficients (ICCs) were used to assess correlation and agreement for daily MVPA and sedentary time estimates from the two methods. Results: Total mean minutes per day spent in MVPA was 12 minutes based on accelerometer data and 26 minutes based on self-reported data $\left(P_{\text {Difference }}<0.01\right)$. Correlation between the methods was fair $(\mathrm{rho}=0.51)$, however agreement was poor $(\mathrm{ICC}=0.33)$. Mean daily time spent sedentary was similar in both methods ( $~ 8.5$ hours), however both correlation and agreement were poor $(\mathrm{rho}=0.19, \mathrm{ICC}=0.16)$.

Conclusions: We found fair correlation but poor agreement between the self-reported and accelerometer-based assessments of MVPA used in this study. For sedentary time, both correlation and agreement between the two methods were poor. Studies of colon cancer survivors using these self-report measures are likely to have a considerable amount of exposure misclassification.

Keywords: Physical activity; sedentary time; validity; accelerometer; colon cancer 


\section{INTRODUCTION}

There is strong evidence that moderate to vigorous-intensity physical activity (MVPA; aerobic activity that requires moderate to hard physical effort and increases breathing and heart rates) has a range of positive physical and psychosocial benefits for cancer survivors [1,2]. Recent research indicates sedentary behaviour (waking activities that require very low energy expenditure and are performed while sitting or reclining [3]) may be associated with poorer physical and psychosocial health in cancer survivors [4]. Given the associations observed between MVPA and sedentary behaviour and a range of outcomes in cancer survivors, it is important to establish the validity of self-reported measures of these behaviours.

Research conducted on the general population has suggested self-reported measures of physical activity and sedentary behaviour are inaccurate when compared with objective measures, such as estimates derived from accelerometers (electronic devices that measure the quantity and intensity of movement) [5-8]. The results of studies concerning the psychometric properties of measurement instruments cannot be systematically generalized to other populations, so it is important to establish the validity of physical activity and sedentary behaviour questionnaires in different populations $[8,9]$. However few studies have compared self-reported and objective measures of physical activity and sedentary behaviour in cancer survivors, a population which is older, has a greater proportion of females, and has more comorbidity than the general population [10].

The few studies that have compared self-reported and objective measures of physical activity in cancer survivors provide mixed results, with some finding acceptable agreement between selfreported and accelerometer-derived physical activity [11-14], and others finding poor agreement 
$[13,15,16]$. Only two studies have investigated agreement between self-reported and accelerometer-derived sedentary behaviour in cancer survivors, with both finding poor to fair agreement $[13,15]$. These studies have generally had small sample sizes $(<100$ participants) and have been conducted in different cancer survivor populations, so it is difficult to draw any conclusions from this research.

The aim of this study was to compare accelerometer-based and self-reported measures of recent moderate- to vigorous-intensity physical activity (MVPA) and sedentary time in colon cancer survivors. Only one previous study has compared self-reported and objective measures of physical activity in colorectal or colon cancer survivors, with the results indicating poor agreement [16].

\section{METHODS}

This study was conducted within the Accurate Measurement of Physical Activity in Colon Cancer Survivors Study (the ACCEL-Colon Study), a cross-sectional study that was conducted in Western Australia (WA) and Alberta, Canada in 2012 [17]. A total of 181 colon cancer survivors (15.1\% response rate), recruited from the WA and Alberta Cancer Registries, wore an Actigraph ${ }^{\circ}$ GT3X+ accelerometer for seven consecutive days and completed a self-administered questionnaire that included questions about recent physical activity and sedentary time. The Actigraph® GT3X+ accelerometer is generally considered to be the 'gold standard' for measuring MVPA. Participants were asked to complete the questionnaire before wearing the accelerometer.

The participants were aged between 27 and 82 (median=65 years), were 12 to 29 months postdiagnosis (median=19 months), were not currently undergoing any adjuvant therapy, and had 
been diagnosed with a Stage I (30\%), II (29\%) or III (40\%) colon cancer. Just over half (54\%) were male, and just over half (51\%) were from Alberta. The participants were predominantly Caucasian (97\%), in a married/common-law/de-facto relationship (78\%), and overweight (44\%) or obese (22\%), Most participants had a post-school qualification (69\%) and were not in the workforce $(61 \%)$.

This study received approval from human research ethics committees at Alberta Health Services, Athabasca University, the WA Department of Health and The University of WA, and all participants gave informed written consent.

\section{Self-reported data}

Recreational physical activity in the past month was assessed using a modified version of the Leisure Score Index of the Godin Leisure-Time Exercise Questionnaire [18]. Participants were asked to report the average number of times per week in the past month that they engaged in a bout of leisure-time physical activity lasting ten or more minutes, and the average duration of these bouts. This was asked for each of vigorous-, moderate- and light-intensity recreational physical activity. The questionnaire defined: vigorous-intensity activities as causing rapid heartbeat and sweating (example activities included aerobics and jogging); moderate-intensity activities as causing light sweating but not being exhausting (examples included brisk walking and pilates); and light-intensity activities as requiring minimal effort and no sweating (examples included easy walking and lawn bowling). As the vast majority of light-intensity physical activity is likely to be performed in the household and occupational domains, which this questionnaire does not measure, light-intensity physical activity was not included in this analysis. 
Data from the vigorous- and moderate-intensity questions were summed to calculate the average time spent per day in MVPA. Bout duration for moderate- and vigorous-intensity physical activity was truncated at 180 minutes [19].

Usual time spent sitting was assessed using the Marshall Domain-Specific Sitting Questionnaire [20]. Participants were asked to report the amount of time they usually spend sitting on weekdays and weekends in each of five domains: travelling to/from work; at work; watching television; using a computer at home; and leisure time (excluding watching television). For both weekdays and weekends the total duration of sitting was truncated at 1080 minutes (18 hours) [21].

\section{Accelerometer data}

The accelerometer data were processed using 60 -second epochs. A cutoff of $<100$ counts per minute (CPM) was used to categorize sedentary time [22], while commonly accepted cutoffs were used to differentiate light-intensity activity (100-1,951 CPM) from MVPA ( $\geq 1952$ CPM) [23]. To be considered valid, days of data collection required $\geq 600$ minutes of wear time and no excessive counts (i.e., $>20,000$ CPM). A period of $\geq 60$ consecutive minutes of zero counts (allowing for $\leq 2$ minutes of observations of $<50 \mathrm{CPM}$ ) was defined as non-wear time [22]. As the self-report method asked respondents to only record bouts of physical activity of $\geq 10$ minutes, only MVPA bouts of $\geq 10$ minutes were taken into account in accelerometer-derived estimates of MVPA. We allowed for one interruption of $\leq 2$ minutes in our definition of a 10 minute bout of MVPA.

\section{Statistical analysis}

We used paired t-tests to compare daily averages of MVPA, moderate-intensity physical activity, vigorous-intensity physical activity and sedentary time obtained from the two methods. Rank- 
order correlation between the methods was assessed using Spearman's rho, while agreement between the methods was assessed using two-way random effects intraclass correlation coefficients (ICC).We also calculated the proportion of participants for whom the estimated daily time on both methods was within five and ten minutes for MVPA, and within one and two hours for sedentary time. For MVPA, we used the kappa-statistic to assess agreement between the methods when classifying participants as meeting or not meeting physical activity guidelines (i.e., $\geq 150$ minutes of MVPA per week),

Exploratory stratified analyses were conducted to determine whether rank-order correlation and/or agreement between the two methods may have been influenced by sex, age (younger/older than 65 years), study location (Australia/Canada), education (high school only/post-school qualification), marital status, body mass index (normal weight/overweight/obese), cancer stage, or comorbidity.

The following cut-off points were used to interpret the rho, ICC and kappa values: $<0.4=$ poor; 0.4-0.59=fair; 0.6-0.74=good; $\geq 0.75=$ excellent. All analyses were conducted using Stata 13.1 (StataCorp, College Station TX).

\section{RESULTS}

The estimated mean minutes per day spent in MVPA was significantly higher for self-reported data than for accelerometer data (23.0 and 12.5 minutes respectively) (Table 1). In $41.4 \%$ of participants the estimated minutes of MVPA per day was within five minutes on the two methods, while estimated daily minutes of MVPA was within 10 minutes for $54.7 \%$ of the participants (data not shown). Rank-order correlation between the methods was fair (rho=0.51), however agreement was poor (ICC=0.33) (Table 2). The proportion of participants meeting 
physical activity guidelines based on self-reported data and accelerometer data was $37.6 \%$ and $24.3 \%$ respectively; agreement between the methods on this measure was poor (kappa=0.32).

Agreement between the two methods for MVPA was slightly stronger among participants who were female, aged 65 years or older, unmarried, had a normal BMI, and had no comorbidity (Table 2). Both correlation and agreement were much stronger for moderate-intensity physical activity than for vigorous-intensity physical activity (Table 2).

Mean sedentary time was similar in both methods ( 8.5 hours and 8.7 hours based on accelerometer and self-reported data respectively) (Table 1). However estimated daily sedentary time was within one hour on the two methods in only $16.6 \%$ of the participants, and within two hours for $42.0 \%$ of the participants. Rank-order correlation and agreement were poor (rho=0.19; ICC $=0.16$ ) (Table 2). Agreement between the methods for sedentary time was slightly stronger among participants who were female, had a post-school qualification, were unmarried, and had no comorbidity (Table 2).

No meaningful effects on the agreement between the methods were observed when we: standardised the accelerometer-derived estimates of MVPA and sedentary time for wear-time; used total MVPA instead of bouts of 10 or more minutes; or used a different cutpoint [24] to determine accelerometer-derived MVPA (data not shown). Using only sedentary bouts of 20 or

more minutes in the accelerometer-derived estimates of sedentary time decreased the correlation and agreement between the methods (rho=0.10, ICC $=0.04$ ).

\section{DISCUSSION}

The results of this study suggest that there is fair correlation and poor agreement between the self-reported and accelerometer-based assessments of MVPA used in this study, but poor 
correlation and agreement for sedentary time. ICCs between the two methods were 0.33 and 0.16 for MVPA and sedentary time respectively, far below the recommended acceptable cut-off of 0.5 [25]. For around $50 \%$ of the participants the difference between self-reported and accelerometer-derived estimates of MVPA and sedentary time was greater than 10 minutes and 2 hours respectively. This divergence suggests that studies of colon cancer survivors using these self-report measures of MVPA and/or sedentary time are likely to have a considerable amount of exposure misclassification, which may lead to biased results.

Our results are consistent with some previous studies in cancer survivors that have also found poor to fair agreement between self-reported and accelerometer-derived physical activity [13, 15, 16], although other studies have found acceptable agreement [11-14]. Two further studies, while not formally assessing validity, have found that cancer survivors over-report MVPA when compared with accelerometer data $[26,27]$. The one previous study conducted among colon or colorectal cancer survivors also found poor to fair agreement between self-reported and accelerometer-derived physical activity [16].

Given the results of previous research, along with the findings of our study, we agree with the recent assertion that accelerometers are arguably the best measures of physical activity and sedentary behaviour in cancer-based studies [28]. This is highly dependent on the study question and practical considerations however [28]. For example, studies relying solely on accelerometerbased physical activity and sedentary time may not be able to provide important information about the context and domain in which physical activity and sedentary time occur, are likely to underestimate time spent in certain physical activities (e.g. swimming and cycling), and are unlikely to be able to obtain measures of strength/resistance training. 
The choice between accelerometers and self-report may also depend on whether a particular study wants to obtain an absolute or relative measure of physical activity. As has been commonly found for self-reported physical activity questionnaires, in this study we found that agreement between the methods was higher when ranking participants from highest to lowest amount of MVPA per day than when quantifying daily MVPA time [8]. This suggests that the self-report method used in this study is able to distinguish between participants with the highest and lowest levels of MVPA, and therefore has acceptable correlation for use in studies relying on relative levels of physical activity (such as those examining associations between MVPA and a particular outcome). This is unlikely to be the case for self-reported sedentary time however, as the poor rank-order correlation and absolute agreement between accelerometer-derived and self-reported estimates of sedentary time observed in this study is consistent with the results of previous studies conducted in the general population $[7,8]$ and cancer survivors $[13,15]$.

There are several methodological factors that may have contributed to the poor agreement found between the two methods. Firstly, while the self-report physical activity questionnaire only asks about physical activity performed in the recreational domain, the accelerometer captures physical activity in all domains. Future research should assess the validity of questionnaires that measure 'total' physical activity in populations of cancer survivors. Secondly, although the accelerometer used in this study is generally considered to be the 'gold standard' when it comes to measuring MVPA, it is likely to underestimate time spent in some activities [28]. For sedentary time, limitations of both methods may have resulted in overestimates of the actual time spent sitting. For self-reported sedentary time it is possible that there was some overlap ('double counting') of sedentary behaviours, so summing these behaviours into a single 'daily sitting time' variable may have lead to an overestimation [20]. With regards to the accelerometer, the 'counts per 
minute' approach used to identify sedentary time likely includes periods of stationary standing as well as sitting, thus resulting in inflated daily sedentary time estimates. A thigh-worn device that can detect body posture and distinguish between sitting and standing, such as the ActivPAL, would have been a better objective measure of sitting time than the accelerometer used in this study. Finally, the two methods did not capture information about physical activity and sedentary time in identical time periods. For MVPA, participants were asked to self-report their average leisure-time physical activity levels over the past month, then to wear the accelerometer for seven days. For sedentary time, the questionnaire asked about 'usual' time spent sitting, and it is possible that the participant did not consider the period in which they wore the accelerometer as 'usual'.

This study has some limitations that should be taken into account. Although we observed some subgroup differences, these stratified analyses were exploratory in nature as our sample was not large enough to draw any firm conclusions regarding differences in agreement by various factors. Previous research indicates that the validity of self-reported measures of physical activity may be influenced by demographic and behavioural factors [6, 29], and more research is needed in this area, particularly for sedentary behaviour. A further limitation is the low response rate and the exclusion of Stage IV cancer survivors. It is possible that people who did not take part in this study, and/or those with Stage IV colon cancer, may have lower levels of physical activity than those who did take part, so our sample may not be representative of the true population of colon cancer survivors. Our results may also not be generalisable to other cancer types.

In summary, we found fair correlation but poor agreement between the self-reported and accelerometer-based assessments of MVPA used in this study conducted among colon cancer survivors. For sedentary time, both correlation and agreement between the two methods were 
poor. Accelerometers are likely to be the best measures of physical activity and sedentary behaviour in colon cancer survivors (particularly in studies wishing to obtain an 'absolute' measure of these behaviours), although this is highly dependent on the aims of the study.

\section{ACKNOWLEDGEMENTS}

Terry Boyle and Brigid Lynch are supported by Early Career Fellowships from the Australian National Health and Medical Research Council (\#1072266 \& \#586727 respectively). Terry Boyle is also supported by a Fellowship from the Canadian Institutes of Health Research (\#300068), a Postdoctoral Fellowship from the Michael Smith Foundation for Health Research (\#5553), and an Honorary Killam Postdoctoral Research Fellowship from The University of British Columbia. Brigid Lynch is also supported by the Victorian Government's Operational Infrastructure Support Program. Jeff Vallance and Kerry Courneya are supported by the Canada Research Chairs program. Jeff Vallance is also supported by a Population Health Investigator Award from Alberta Innovates - Health Solutions. We would like to acknowledge Parneet Sethi and Dr. Elisabeth Winkler for their assistance in data processing and analyses.

\section{CONFLICT OF INTEREST STATEMENT}

None of the authors have any conflicts of interest to declare 
Table 1. Accelerometer-derived and self-reported estimates of daily moderate-to-vigorous intensity physical activity (MVPA) and sedentary time in colon cancer survivors Mean Minutes of Physical Activity (95\% CI)

\begin{tabular}{llll}
\hline & Moderate- & Vigorous- & Mean Hours of Sedentary \\
MVPA & Intensity & Intensity & Time (95\% CI)
\end{tabular}

\begin{tabular}{lllll}
\hline Accelerometer & $12.5(9.8-15.1)$ & $11.5(9.0-14.0)$ & $0.5(0.2-0.8)$ & $8.7(8.5-9.0)$ \\
Self-Report & $23.0(18.5-27.5)$ & $17.1(13.3-20.9)$ & $5.9(3.7-8.1)$ & $8.5(8.0-9.0)$ \\
Mean Difference & $-10.6(-14.8,-$ & $-5.5(-9.2,-1.9)$ & $-5.4(-7.6,-3.3)$ & $0.3(-0.3,0.8)$ \\
& $6.4)$ & & & \\
$\boldsymbol{P}_{\text {Difference }}$ & $<0.01$ & $<0.01$ & $<0.01$ & 0.33
\end{tabular}


Table 2. Correlation and agreement between accelerometer-derived and self-reported estimates of moderate-to-vigorous intensity physical activity (MVPA) and sedentary time in colon cancer survivors, overall and by subgroup

\begin{tabular}{|c|c|c|c|c|}
\hline & \multicolumn{2}{|c|}{ MVPA } & \multicolumn{2}{|c|}{ Sedentary Time } \\
\hline & rho & ICC $(95 \%$ CI $)$ & rho & ICC $(95 \% \mathrm{CI})$ \\
\hline All Participants & 0.51 & $0.33(0.18-0.46)$ & 0.19 & $0.16(0.01-0.30)$ \\
\hline Moderate-Intensity PA only & 0.44 & $0.34(0.20-0.46)$ & - & - \\
\hline Vigorous Intensity PA only & 0.17 & $0.03(-0.10-0.16)$ & - & - \\
\hline \multicolumn{5}{|l|}{ Sex } \\
\hline Male & 0.56 & $0.25(0.06-0.42)$ & 0.09 & $0.11(-0.09-0.30)$ \\
\hline Female & 0.46 & $0.43(0.24-0.59)$ & 0.25 & $0.20(-0.02-0.40)$ \\
\hline \multicolumn{5}{|l|}{ Age } \\
\hline$<65$ years & 0.45 & $0.22(0.02-0.41)$ & 0.19 & $0.18(-0.03-0.38)$ \\
\hline$\geq 65$ years & 0.55 & $0.40(0.20-0.57)$ & 0.21 & $0.15(-0.04-0.34)$ \\
\hline
\end{tabular}

\section{Location}
Alberta, Canada
0.44
$0.33(0.13-0.49)$
0.21
$0.19(-0.02-0.38)$
Western Australia
0.57
$0.33(0.13-0.51)$
0.15
$0.11(-0.09-0.31)$

\section{Education Level}

High School Only

0.39

$0.35(0.10-0.55)$

0.11

$0.05(-0.22-0.31)$

Post-school qualification

0.53

$0.31(0.13-0.47)$

0.22

$0.22(0.05-0.38)$

\section{Marital Status}

Not Married

0.53

$0.45(0.16-0.66)$

0.18

$0.25(-0.08-0.52)$

Married/De-facto

0.50

$0.27(0.11-0.42)$

0.18

$0.13(-0.03-0.29)$ 


\section{Body Mass Index}

$\begin{array}{lcccc}\text { Normal } & 0.47 & 0.48(0.26-0.66) & 0.21 & 0.14(-0.10-0.37) \\ \text { Overweight } & 0.57 & 0.27(0.06-0.46) & 0.16 & 0.15(-0.08-0.36) \\ \text { Obese } & 0.46 & 0.14(-0.14-0.41) & 0.12 & 0.12(-0.21-0.42) \\ \text { Stage } & & & & \\ 1 & 0.60 & 0.43(0.18-0.62) & 0.09 & 0.06(-0.23-0.33) \\ 2 & 0.42 & 0.19(-0.07-0.42) & 0.11 & 0.13(-0.15-0.38) \\ 3 & 0.51 & 0.36(0.13-0.55) & 0.26 & 0.22(-0.02-0.43)\end{array}$

\section{Self-Reported Comorbidity}

None

One or more
0.47

$0.45(0.25-0.62)$

0.30

$0.24(0.01-0.45)$

$0.53 \quad 0.25(0.06-0.42) \quad 0.11$

$0.10(-0.09-0.28)$ 


\section{REFERENCES}

1. Rock CL, Doyle C, Demark-Wahnefried Wet al (2012) Nutrition and physical activity guidelines for cancer survivors. CA Cancer J Clin 62: 242-274

2. Schmitz KH, Courneya KS, Matthews Cet al (2010) American College Of Sports Medicine roundtable on exercise guidelines for cancer survivors. Med Sci Sports Exerc 42: $1409-1426$

3. Sedentary Behaviour Research Network (2012) Letter to the editor: Standardized use of the terms "sedentary" and "sedentary behaviours". Appl Physiol Nutr Metab 37: 540-542

4. Lynch BM, Dunstan DW, Vallance JK, Owen N (2013) Don't take cancer sitting down. Cancer 119: 1928-1935

5. Prince S, Adamo K, Hamel Met al (2008) A comparison of direct versus self-report measures for assessing physical activity in adults: A systematic review. Int J Behav Nutr Phys Act 5: 56

6. Dyrstad SM, Hansen BH, Holme IM, Anderssen SA (2014) Comparison of self-reported versus accelerometer-measured physical activity. Med Sci Sports Exerc 46: 99-106

7. Healy GN, Clark BK, Winkler EAHet al (2011) Measurement of adults' sedentary time in population-based studies. Am J Prev Med 41: 216-227

8. Helmerhorst HJ, Brage S, Warren J, Besson H, Ekelund U (2012) A systematic review of reliability and objective criterion-related validity of physical activity questionnaires. Int $\mathbf{J}$ Behav Nutr Phys Act 9: 103

9. van Poppel MNM, Chinapaw MJM, Mokkink LB, van Mechelen W, Terwee CB (2010) Physical activity questionnaires for adults: A systematic review of measurement properties. Sports Med 40: 565-600 
10. Mao JJ, Armstrong K, Bowman MAet al (2007) Symptom burden among cancer survivors: Impact of age and comorbidity. The Journal of the American Board of Family Medicine 20: 434-443

11. Jovanovic JL, Hughes DC, Baum GPet al (2011) Accelerometry and self-report in sedentary populations. Am J Health Behav 35: 71-80

12. Su C-C, Lee K-D, Yeh C-H, Kao C-C, Lin C-C (2014) Measurement of physical activity in cancer survivors: A validity study. J Cancer Surviv 8: 205-212

13. Johnson-Kozlow M, Sallis J, Gilpin E, Rock C, Pierce J (2006) Comparative validation of the IPAQ and the 7-day PAR among women diagnosed with breast cancer. Int J Behav Nutr Phys Act 3: 7

14. Johnson-Kozlow M, Rock CL, Gilpin EA, Hollenbach KA, Pierce JP (2007) Validation of the WHI Brief Physical Activity Questionnaire among women diagnosed with breast cancer. Am J Health Behav 31: 193-202

15. Liu R, Buffart L, Kersten MJet al (2011) Psychometric properties of two physical activity questionnaires, the AQUAA and the PASE, in cancer patients. BMC Med Res Methodol 11: 30

16. Pinto BM, Papandonatos GD, Goldstein MG, Marcus BH, Farrell N (2013) Home-based physical activity intervention for colorectal cancer survivors. Psychooncology 22: 54-64

17. Vallance JK, Boyle T, Courneya KS, Lynch BM (2014) Associations of objectivelyassessed physical activity and sedentary time with health-related quality of life among colon cancer survivors. Cancer: Published Ahead of Print June 42014

18. Godin G, Shephard RJ (1985) A simple method to assess exercise behavior in the community. Can J Appl Sport Sci 10: 141-146 
19. IPAQ Research Committee (2005) Guidelines for data processing and analysis of the International Physical Activity Questionnaire (IPAQ)-Short and Long Forms. http://www.ipaq.ki.se/scoring.pdf. Accessed May 14, 2014

20. Marshall AL, Miller YD, Burton NW, Brown WJ (2010) Measuring total and domainspecific sitting: A study of reliability and validity. Med Sci Sports Exerc 42: 1094-1102

21. Gardiner PA, Healy GN, Eakin EGet al (2011) Associations between television viewing time and overall sitting time with the metabolic syndrome in older men and women: The Australian Diabetes Obesity and Lifestyle Study. J Am Geriatr Soc 59: 788-796

22. Matthews CE, Chen KY, Freedson PSet al (2008) Amount of time spent in sedentary behaviors in the United States, 2003-2004. Am J Epidemiol 167: 875-881

23. Freedson PS, Melanson E, Sirard J (1998) Calibration of the Computer Science and Applications, Inc. accelerometer. Med Sci Sports Exerc 30: 777-781

24. Sasaki JE, John D, Freedson PS (2011) Validation and comparison of Actigraph activity monitors. J Sci Med Sport 14: 411-416

25. Terwee CB, Mokkink LB, van Poppel MNM et al (2010) Qualitative attributes and measurement properties of physical activity questionnaires: A checklist. Sports Med 40: $525-537$

26. Broderick JM, Hussey J, Kennedy MJ, O’Donnell DM (2014) Testing the 'teachable moment' premise: Does physical activity increase in the early survivorship phase? Support Care Cancer 22: 989-997

27. Broderick JM, Guinan E, Kennedy MJet al (2013) Feasibility and efficacy of a supervised exercise intervention in de-conditioned cancer survivors during the early survivorship phase: The PEACH trial. J Cancer Surviv 7: 551-562 
28. Broderick JM, Ryan J, O’Donnell DM, Hussey J (2014) A guide to assessing physical activity using accelerometry in cancer patients. Support Care Cancer 22: 1121-1130

29. Bull FC, Maslin TS, Armstrong T (2009) Global Physical Activity Questionnaire (GPAQ): Nine country reliability and validity study. J Phys Act Health 6: 790-804 